\title{
Avaliação pós-operatória comparativa dos pacientes submetidos ao tratamento cirúrgico das luxações acromioclaviculares aguda versus crônica*
}

\section{Postoperative Comparative Evaluation of Patients Undergoing Surgical Treatment for Acute Versus Chronic Acromioclavicular Dislocations}

\author{
Thiago Medeiros Storti ${ }^{1,20}$ Leony Batista de Paula ${ }^{1}$ Carolina Simionatto ${ }^{1}$ João Eduardo Simionatto ${ }^{1}$ \\ Rafael Salomon Silva Faria ${ }^{1}$ Alexandre Firmino Paniago ${ }^{1}$ \\ ${ }^{1}$ Instituto do Ombro de Brasília, Brasília, DF, Brasil \\ ${ }^{2}$ Grupo de Ombro, Instituto de Pesquisa e Ensino HOME (IPE HOME), \\ Endereço para correspondência Thiago Medeiros Storti, Quadra 102 \\ norte, Praça Perdiz, Lote 05, Condomínio Residencial Matisse Antares, \\ Brasília, DF, Brasil \\ Águas Claras, DF, 71907-000, Brasil (e-mail: thiago_storti@hotmail.com).
}

Rev Bras Ortop 2021;56(1):98-103.

\begin{abstract}
Resumo
Objetivos Avaliar e comparar os resultados do tratamento cirúrgico das luxações acromioclaviculares (LACs) aguda e crônica, definindo o plano terapêutico mais eficaz. Métodos Estudo retrospectivo realizado com 30 pacientes operados entre 2011 e 2018 para LAC tipos III e $\mathrm{V}$, separados de acordo com a classificação temporal em subgrupo agudo ( $<3$ semanas; subgrupo I) e subgrupo crônico ( $>3$ semanas; subgrupo II). Todos os pacientes foram submetidos a avaliação pós-cirúrgica com protocolo padronizado composto por dados epidemiológicos, funcionais e radiográficos.

Resultados No subgrupo I, a pontuação na escala visual analógica (EVA) foi de 1,10, o escore de Constant-Murley foi de 92,3, e o escore da University of California at Los Angeles (UCLA) foi de 33,5. A distância coracoclavicular (CC) foi de $11,0 \mathrm{~mm}$, e o aumento do espaço

Palavras-chave

- articulação acromioclavicular/ lesões

- articulação acromioclavicular/ cirurgia

- luxações articulares

- ligamentos articulares CC foi em média menor do que $8,9 \%$ em relação ao ombro contralateral. No subgrupo II, a EVA foi de 1,11, o escore de Constant-Murley foi de 94,2, e o da UCLA, 32,4. A distância CC foi de $13,8 \mathrm{~mm}$, sendo o aumento do espaço CC de $22,9 \%$ em relação ao contralateral. Conclusão Apesar de não ter havido diferença significativa entre os quesitos avaliados, houve uma tendência de o subgrupo agudo apresentar distância CC $(p=0,098)$ e percentual de aumento da distância CC $(p=0,095)$ menor do que o subgrupo crônico. Assim, é interessante que o tratamento cirúrgico seja realizado nas primeiras três semanas após o trauma, para tentar evitar essa tendência. Nos casos em que não for possível realizar o tratamento na fase aguda, a técnica de Weaver Dunn modificada apresenta bons resultados clínicos e funcionais.
\end{abstract}

Trabalho realizado no Hospital Ortopédico e Medicina Especializada (HOME), Brasília, DF, Brasil.

recebido

30 de Setembro de 2019

aceito

20 de Abril de 2020

Publicado online

Setembro 30, 2020
DOI https://doi.org/ $10.1055 / \mathrm{s}-0040-1713761$ ISSN 0102-3616. (c) 2020. Sociedade Brasileira de Ortopedia e Traumatologia. All rights reserved.

This is an open access article published by Thieme under the terms of the Creative Commons Attribution-NonDerivative-NonCommercial-License, permitting copying and reproduction so long as the original work is given appropriate credit. Contents may not be used for commercial purposes, or adapted, remixed, transformed or built upon. (https://creativecommons.org/ licenses/by-nc-nd/4.0/)

Thieme Revinter Publicações Ltda., Rua do Matoso 170, Rio de Janeiro, RJ, CEP 20270-135, Brazil 
Abstract

\section{Keywords}

- acromioclavicular joint/injuries

- acromioclavicular joint/surgery

- joint dislocations

- ligaments, articular
Objectives The present study evaluates and compares the surgical treatment of acute and chronic acromioclavicular dislocations (ACDs) to define the most effective therapeutic plan.

Methods A retrospective study consisting of 30 patients submitted to the surgical treatment of types III and V ACDs between 2011 and 2018; the subjects were separated according to a temporal classification in acute ( $<3$ weeks; subgroup I) and chronic ( $>$ 3 weeks; subgroup II) subgroups. All patients underwent a postsurgical evaluation with a standardized protocol containing epidemiological, functional, and radiological data. Results subgroup I presented a visual analog scale (VAS) score of 1.10, a ConstantMurley score of 92.3, and a University of California at Los Angeles (UCLA) Shoulder Rating score of 33.5. The coracoclavicular (CC) distance was of $11.0 \mathrm{~mm}$, and the average increase in CC space was lower than $8.9 \%$ compared to the contralateral shoulder. In subgroup II, the VAS score was of 1.11, the Constant-Murley score was of 94.2, and the UCLA score was of 32.4. The CC distance was of $13.8 \mathrm{~mm}$, with a $22.9 \%$ increase in CC space compared to the contralateral side.

Conclusion Although there was no significant difference between the evaluated items, subgroup I tended to present a lower CC distance $(p=0.098)$ and a lower percentage increase in CC distance $(p=0.095)$ compared to subgroup II. Thus, the surgical treatment must be performed within three weeks after the trauma to try to avoid such trend. If the acute treatment is not possible, the modified Weaver Dunn technique has good clinical and functional outcomes.

\section{Introdução}

A articulação diartrodial entre a face medial do acrômio e a lateral da clavícula é denominada articulação acromioclavicular (AC). A estabilidade anteroposterior (AP) da articulação é promovida pelos ligamentos ACs, que, por sua vez, são espessamentos da cápsula articular, sendo a porção superior a mais forte. A estabilidade superoinferior é mantida pelos ligamentos coracoclaviculares (CCs) e pelos ligamentos trapezoide e conoide. $^{1}$

Fukuda et al., ${ }^{2}$ identificaram em seus testes que os ligamentos ACs, em pequenos deslocamentos, foram os limitadores primariamente nas translações posterior e superior. Já em grandes deslocamentos, o ligamento conoide foi o responsável pela limitação primária da translação superior, e o ligamento trapezoide promoveu resistência à compressão da articulação AC. ${ }^{2}$

Tipicamente, as lesões na articulação AC resultam de trauma direto no ombro ocasionado por quedas e esportes de contato com o braço em posição aduzida. A força desvia o acrômio inferiormente, enquanto a clavícula se mantém na posição anatômica. Isso resulta em graus variáveis de lesão dos ligamentos AC e CC. ${ }^{3}$

Williams et al., ${ }^{4}$ com base no trabalho de Tossy et al., ${ }^{5}$ desenvolveram um sistema de classificação amplamente aceito, baseado na gravidade anatômica das lesões, dividindo-as em seis tipos. Além disso, as lesões ACs podem ser divididas em agudas ( $<3$ semanas) e crônicas ( $>3$ semanas). ${ }^{6,7}$

0 tratamento não cirúrgico tem sido recomendado para as lesões dos tipos I e II pela maioria dos autores. ${ }^{1,8,9}$ Nas lesões do tipo III, o tratamento é controverso, e vários autores apresentam séries de casos tratados de maneira conservadora com resultados entre bons e excelentes. ${ }^{10,11}$ Entretanto, outros autores apresentam casos com dor e outros sintomas residuais. ${ }^{12,13}$ Para tentar maximizar os resultados positivos, muitos autores têm defendido a indicação de reparo cirúrgico nos casos de pacientes jovens e ativos. ${ }^{14,15}$

Nas lesões dos tipo IV, V e VI o tratamento consagrado é o cirúrgico. ${ }^{1,3,9,14}$ Múltiplas técnicas foram descritas para o tratamento cirúrgico, mas não há evidências da superioridade de alguma delas em comparação com as outras. $O$ único consenso é que cinco elementos-chave devem ser atingidos independente da abordagem: redução anatômica, reconstrução ou reparo direto dos ligamentos CCs, proteção dos ligamentos CCs, reparo da fáscia deltotrapezoidal, e, nos casos de lesões crônicas, ressecção distal da clavícula. ${ }^{3}$

O objetivo deste trabalho é analisar os resultados do tratamento das luxações acromioclaviculares (LACs) aguda e crônica para identificar o melhor momento para o tratamento cirúrgico e definir um plano terapêutico mais eficaz.

\section{Material e Métodos}

Foi realizado um estudo transversal retrospectivo com 39 casos de cirurgias para LAC realizados entre 2011 e 2018, em 2 hospitais privados. Pelo longo tempo de seguimento e perfil dos pacientes, apenas 30 retornaram para avaliação. Todos foram avaliados radiograficamente nas incidências AP e Zanca, incluindo os dois ombros, e na incidência axilar. Dos pacientes acompanhados, 28 foram diagnosticados no 
Tabela 1 Variáveis clínicas segundo o subgrupo

\begin{tabular}{|l|l|l|l|l|}
\hline \multirow{2}{*}{ Variável clínica } & \multicolumn{2}{|l|}{ Subrupo agudo $(\mathbf{n}=\mathbf{2 1})$} & \multicolumn{2}{l|}{ Subrupo crônico $(\mathbf{n}=\mathbf{9})$} \\
\cline { 2 - 5 } & Média & Desvio padrão & Média & Desvio padrão \\
\hline Idade (anos) & 40,7 & 13,1 & 42,1 & 14,6 \\
\hline Tempo até a cirurgia (dias) & 4,5 & 4,0 & 424 & 462 \\
\hline Tempo até o retorno ao trabalho (dias) ${ }^{\mathrm{a}}$ & 73,0 & 49,8 & 78,1 & 49,1 \\
\hline Tempo até o retorno ao esporte (meses) $)^{\mathrm{b}}$ & 5,3 & 2,8 & 7,50 & 5,01 \\
\hline Lateralidade & & & & \\
\hline Direita & $15(71,4 \%)$ & $6(66,7 \%)$ & \\
\hline Esquerda & $6(28,6 \%)$ & $3(33,3 \%)$ \\
\hline
\end{tabular}

Notas: Os dados numéricos foram expressos pela média e desvio padrão.

${ }^{a} O$ tempo até o retorno ao trabalho apresentou perda de registro, ou não se aplica nos 2 grupos, agudo e crônico $(n=19$ versus $n=7)$.

${ }^{\mathrm{b}} \mathrm{O}$ tempo até o retorno ao esporte apresentou perda de registro, ou não se aplica nos 2 grupos, agudo e crônico $(\mathrm{n}=17$ versus $\mathrm{n}=8)$.

momento da cirurgia com LAC de grau $\mathrm{V}$, e os outros 2 pacientes foram diagnosticados com LAC de grau III.

Dos 30 pacientes, 21 apresentavam lesões agudas (subgrupo I), e 9, lesões crônicas (subgrupo II). O subgrupo I foi operado em média 4,5 dias após o trauma, enquanto o subgrupo II foi operado em média 424 dias após o trauma. O sexo masculino foi o mais prevalente $(96,6 \%)$. A idade média foi de 40,7 anos no subgrupo I, e de 42,1 anos no subgrupo II, com o lado direito sendo o mais acometido, em $71,4 \%$ do subgrupo I, e em $66,7 \%$ do subgrupo II (-Tabela $\mathbf{1}$ ).

Todos os pacientes foram reavaliados por um mesmo examinador, sendo submetidos a avaliação pós-cirúrgica a partir de protocolo padronizado composto do escore da University of California at Los Angeles (UCLA), do escore de Constant-Murley, da escala visual analógica (EVA), de dados epidemiológicos, e da avaliação de força de modo comparativo contralateral utilizando um dinamômetro digital. Após a avaliação, realizamos exames radiográficos para a determinação do deslocamento residual no ombro operado, comparando a distância CC à do ombro contralateral, por meio das incidências AP e Zanca, incluindo os dois ombros, e na incidência axilar.

O desenho do estudo foi submetido e aprovado pelo comitê de ética local, sob o CAAE 95443218.4.0000.0023.

\section{Metodologia Estatística}

$\mathrm{Na}$ análise descritiva, foram feitas tabelas que apresentam os dados observados expressos pela média e desvio padrão para dados numéricos, e pela frequência e porcentagem para os dados categóricos.

A análise inferencial foi feita para a comparação entre os subgrupos (agudo e crônico), e foram usados o teste de MannWhitney para dados numéricos, e o teste exato de Fisher para os dados categóricos. A comparação entre dados pareados foi realizada usando-se o teste dos postos sinalizados de Wilcoxon. O coeficiente de correlação de Spearman foi utilizado para analisar associação entre variáveis numéricas.

Foram utilizados métodos não paramétricos, pois nenhum dos dados apresentou distribuição normal (gaus- siana), devido à rejeição da hipótese nula de normalidade segundo o teste de Shapiro-Wilk, em pelo menos um grupo e/ ou momento. $O$ critério de determinação de significância adotado foi o nível de $5 \%$. A análise estatística foi processada pelo software estatístico SAS System (SAS Institute, Inc., Cary, North Carolina, EUA), versão 6.11.

\section{Técnica Cirúrgica}

O tratamento para lesões agudas preconizado pelos autores é a técnica com uso de âncoras de sutura associada à fixação transarticular com fio de Kirschner descrita por Phemister. ${ }^{16}$ As vantagens são a pequena incisão e dissecção limitada na região acima do coracoide, não sendo necessário nenhum instrumento abaixo deste, o que minimiza o risco de lesão neurovascular.

Com o paciente na posição de cadeira de praia, anestesiado com bloqueio interescalênico, o braço e ombro são preparados. Uma incisão de $5 \mathrm{~cm}$ é feita abaixo da clavícula, no nível do processo coracoide. O subcutâneo é dissecado até a exposição da fáscia deltotrapezoidal. É realizada então uma incisão de medial para lateral seguindo a curvatura da clavícula até a exposição óssea.

Realiza-se dissecção romba até a exposição da base dorsal do processo coracoide. Após exposição satisfatória, são utilizadas 2 âncoras de sutura ${ }^{\circ} 5$ com 2 fios de sutura inabsorvíveis $n^{\circ} 2$ (Fiberwire, Arthrex, Naples, Flórida, EUA). Uma broca de 3,2 mm é usada para fazer 2 orifícios na clavícula, sendo um mais posterior, a $3,5 \mathrm{~cm}$ da articulação $\mathrm{AC}$, e o outro mais anterior, a $2,5 \mathrm{~cm}$ da articulação AC. ${ }^{17,18}$

É realizada a hiperredução da luxação, e um fio de Kirschner de 2,0 mm é transfixado pela articulação AC. Seu posicionamento é confirmado por escopia. Após a redução, cada fio de sutura é amarrado separadamente. A fáscia deltotrapezoidal é reparada, e o subcutâneo e a pele são suturados. Os fios de Kirschner são dobrados, e permanecem sob a pele.

O tratamento das luxações crônicas foi feito por meio da técnica de Weaver-Dunn modificada. O posicionamento e preparo do paciente são iguais ao procedimento anterior, 
também sendo utilizadas duas âncoras de sutura no coracoide. É realizada uma incisão medial à articulação AC de $5 \mathrm{~cm}$ a $7 \mathrm{~cm}$ em direção ao processo coracoide. A fáscia deltotrapezoidal é identificada e incisada. Então, faz-se o descolamento periosteal do trapézio e do deltoide. Por meio de dissecção romba, o ligamento coracoacromial é identificado e desinserido em sua inserção anteroinferior no acrômio. A extremidade lateral da clavícula é excisada em torno de $1,0 \mathrm{~cm}$ a $1,5 \mathrm{~cm}$ da borda lateral.

É feita então a redução da clavícula, e passados 1 ou 2 fios de Kirschner transfixando a articulação AC. São feitos dois orifícios na cortical superior da clavícula, e a extremidade do ligamento coracoacromial é reparada e amarrada passando pelos orifícios da clavícula, de forma que o ligamento é projetado para o canal medular. Os fios das âncoras são amarrados na clavícula, a fáscia deltotrapezoidal é reparada, e o subcutâneo e a pele são suturados. Assim como nos casos agudos, os fios de Kirschner são dobrados e permanecem sob a pele.

\section{Pós-operatório}

Nos dois grupos, o braço foi mantido em uma tipoia americana de três pontos por seis semanas. Ao final da sexta semana, o fio de Kirschner foi removido cirurgicamente, e a mobilização foi permitida. Fisioterapia motora foi iniciada a partir de então, para ganho de amplitude de movimento e alongamento. $\mathrm{O}$ fortalecimento muscular foi liberado após o terceiro mês de pós-operatório, e o retorno à prática esportiva, após o quinto mês.

\section{Resultados}

Na avaliação dos scores funcionais, a EVA média do subgrupo I foi de 1,10 (desvio padrão [DP]: 1,61), e a do subgrupo II foi de 1,11 (DP: 2,09). O escore de Constant-Murley teve média de 92,3 (DP: 7,1) no subgrupo I, e, no subgrupo II, de 94,2 (DP: 6,9). Já o escore da UCLA apresentou no subgrupo I média de 33,5 (DP: 2,2), e, no subgrupo II, de 32,4 (DP: 4,9).Observouse que não houve diferença significativa, no nível de $5 \%$, nos scores funcionais (-Tabela 2 ).

$\mathrm{Na}$ avaliação de força, a média de abdução no braço operado do subgrupo I foi de 11,1 kgf (DP: 5,4), com delta da força da variação relativa do ombro operado em relação ao contralateral de $-2,94 \%$. No subgrupo II, a média no braço operado foi $11,5 \mathrm{kgf}$ (DP: 3,4 ) com delta da força de $-7,54 \%$.

A força média da rotação medial no subgrupo I foi de 15,9 kgf (DP: 8,9) no braço operado, com delta da força de $-3,23 \%$. No subgrupo II, a média foi de $14,2 \mathrm{kgf}$ (DP: 3,8 ) no braço operado, com delta da força de $-1,37 \%$.

A força média da rotação lateral no subgrupo I foi de 11,2 kgf (DP: 5,1) no braço operado, com delta da força de $-7,21 \%$. No subgrupo II, a média foi de $10,5 \mathrm{kgf}$ (DP: 2,9 ) no braço operado, com delta da força de $-4,68 \%$. Nas variáveis de força, também se observou que não houve diferença significativa no nivel de 5\% (-Tabela 2 ).

A média da distância CC no subgrupo I foi de $11,0 \mathrm{~mm}$ (DP: 4,0), e, no subgrupo II, a média foi de $13,8 \mathrm{~mm}$ (DP: 4,1). No subgrupo I, 38,09\% dos pacientes apresentaram subluxação da articulação AC com aumento do espaço CC em relação ao

Tabela 2 Variáveis funcionais e de força segundo o subgrupo

\begin{tabular}{|l|l|l|l|l|}
\hline Variáveis & \multicolumn{2}{|l|}{ Subgrupo agudo (n=21) } & \multicolumn{2}{l|}{ Subgrupo crônico (n=9) } \\
\hline Funcional & Média & Desvio padrão & Média & Desvio padrão \\
\hline EVA (pontos) & 1,10 & 1,61 & 1,11 & 2,09 \\
\hline Escore de Constant-Murley (pontos) & 92,3 & 7,1 & 94,2 & 6,9 \\
\hline Escore da UCLA (pontos) & 33,5 & 2,2 & 32,4 & 4,9 \\
\hline Distância CC (mm) & 11,0 & 4,0 & 13,8 & 4,1 \\
\hline \% de aumento da distância & 8,9 & 14,2 & 22,9 & 24,1 \\
\hline Força do ombro operado & Média & Desvio padrão & Média & Desvio padrão \\
\hline Força de abdução (kg) & 11,1 & 5,4 & 11,5 & 3,4 \\
\hline Força de rotação medial (kg) & 15,9 & 8,9 & 14,2 & 3,8 \\
\hline Força de rotação lateral (kg) & 11,2 & 5,1 & 10,5 & 2,9 \\
\hline Força do ombro contralateral & Média & Desvio padrão & Média & Desvio padrão \\
\hline Força de abdução (kg) & 11,7 & 5,4 & 12,5 & 3,8 \\
\hline Força de rotação medial (kg) & 15,6 & 9,1 & 14,6 & 4,5 \\
\hline Força de rotação lateral (kg) & 12,2 & 5,7 & 11,4 & 4,4 \\
\hline Delta relativo da força (\%) & & & & \\
\hline Delta da força de abdução (\%) & $-2,94$ & & $-7,54$ & \\
\hline Delta da força de rotação medial (\%) & 3,23 & $-1,37$ & \\
\hline Delta da força de rotação lateral (\%) & $-7,21$ & $-4,68$ & \\
\hline
\end{tabular}

Abreviaturas: CC, coracoclavicular; EVA, escala visual analógica; UCLA, University of California at Los Angeles.

Notas: Os dados numéricos foram expressos pela média e desvio padrão. O delta da força corresponde à variação relativa do ombro operado em relação ao contralateral: (operado-contralateral)/contralateral x 100. 
ombro contralateral em média de 8,9\% (DP: 14,2), enquanto, no subgrupo II, $66,66 \%$ dos pacientes apresentaram subluxação com ascensão média de 22,9\% (DP: 24,1). Verificamos uma tendência de o subgrupo agudo apresentar distância CC $(p=0,098)$ e porcentagem de aumento $(p=0,095)$ menor do que o subgrupo crônico. Observou-se ainda que não houve correlação significativa, no nível de $5 \%$, entre o percentual de aumento da distância com os parâmetros funcionais e de força, tanto na amostra total quanto nos subgrupos agudo e crônico.

O tempo para retorno ao trabalho foi em média de 73 dias para subgrupo I, e de 78,1 dias para o subgrupo II. Já com relação ao retorno à prática esportiva, no subgrupo I ele ocorreu em média com 5,3 meses (DP: 2,8), contra 7,5 meses (DP: 5,01) no subgrupo II.

Os resultados funcionais e de força foram descritos na

-Tabela 2.

\section{Discussão}

Não existe consenso na literatura atual sobre qual é a melhor técnica cirúrgica para o tratamento tanto das LACS crônicas quanto das agudas. Publicações científicas têm apresentado os resultados de várias formas de tratamento dessas lesões, mas poucas comparam os resultados entre as técnicas utilizadas para cada um desses casos. Em nosso trabalho, avaliamos os resultados do tratamento cirúrgico de luxações agudas e crônicas, buscando compará-los.

Ambos os grupos foram submetidos a técnicas cirúrgicas que seguem os cinco elementos-chave do tratamento cirúrgico preconizados por Li et al. ${ }^{3}$ : redução anatômica, reconstrução ou reparo direto dos ligamentos CC, proteção dos ligamentos CC, reparo da fáscia deltotrapezoidal, e, nos casos de lesões crônicas, ressecção distal da clavícula.

Diferente do trabalho de Von Heideken et al., ${ }^{19}$ que encontraram diferença estatisticamente significativa no escore de Constant-Murley (91 no grupo agudo versus 85 no grupo crônico), e do de Rolf et al., ${ }^{20}$ que também relataram resultados clínicos e funcionais inferiores no grupo de reconstrução tardia $(87,17$ versus 78,10$)$, obtivemos pontuações dos escores de Constant-Murley e da UCLA sem diferença estatística entre o grupo 1 e o grupo 2 .

Tauber et al., ${ }^{21}$ durante a avaliação da EVA, obtiveram em média resultado de 2,3 pontos, semelhante ao encontrado por Hegazy et al. ${ }^{22}$ em sua série (média de 1 ponto), o que condiz com nossa avaliação da EVA, que apresentou média de 1,10 pontos no grupo agudo, e de 1,11 pontos no grupo crônico, não apresentando significância estatística.

$\mathrm{Na}$ avaliação pelo escore da UCLA, obtivemos média de 33,5 pontos (DP: 2,2 ) no grupo 1 , sendo os valores considerados bons/excelentes ( $>27$ pontos) em 95,23\% dos casos. No grupo 2, a média foi de 32,4 pontos (DP: 4,9 ) sendo o resultado considerado bom/excelente em $88,8 \%$ dos casos, não existindo diferença estatisticamente significativa entre os grupos. Esses resultados são semelhantes aos vistos na literatura nacional, com 92,8\% de casos bons/excelentes vistos por Molin et al., ${ }^{23}$ e 95,2\% vistos por Scandiuzzi et al., 24
As complicações estiveram presentes em $43,3 \%$ de nossos pacientes, percentagem similar à encontrada em outros estudos, como o de Ferreira Neto et al. ${ }^{25}$ (40,9\%) e Neviaser, ${ }^{26}$ que relatou 39\%. No subgrupo I, infecção superficial ocorreu em 14,21\% dos casos, enquanto no subgrupo II ela ocorreu em $11,11 \%$. Todos os casos foram tratados com antibioticoterapia oral e curativos diários, não ocorrendo deiscência da sutura da pele, e sem repercussões clínicas. Outra complicação observada foi a migração lateral do fio de Kirschner AC, presente em um paciente do subgrupo I (4,76\%), sendo o paciente tratado com a retirada do material de síntese, não havendo outras complicações. Dor residual foi queixa de $14,28 \%$ dos pacientes do subgrupo I, e de $11,11 \%$ do subgrupo II.

A proeminência da clavícula foi queixa de 4,76\% dos pacientes do subgrupo I, e de $22.2 \%$ do subgrupo 2 . 0 aumento do espaço CC acima de $12 \mathrm{~mm}$, avaliado de forma radiográfica, foi observado em média em $8,9 \%$ dos pacientes do subgrupo I (DP: 14,2); no subgrupo, 2 a média foi de 22,9\% (DP: 24,1). Apesar de não ter havido diferença significativa no nível de $5 \%$, houve uma tendência de o subgrupo agudo apresentar distância CC $(p=0,098)$ e percentual de aumento da distância CC em relação ao contralateral $(p=0,095)$ menor do que o subgrupo crônico.

Constatamos que, apesar da alta incidência desse desvio como complicação, não houve repercussão final no nível de satisfação dos pacientes, e nenhum deles evoluiu com discinesia escapular, o que corrobora os trabalhos encontrados na literatura, em que a redução anatômica nem sempre é necessária para restaurar a a função adequada do ombro, e a perda na redução parece não influenciar significativamente o resultado. ${ }^{12,27,28}$

No total, 28 pacientes (93,3\%) ficaram satisfeitos com o tratamento, não existindo diferença estatisticamente significativa entre os grupos, o que sugere, como na literatura, ${ }^{13}$ que não há relação entre os achados clínicos e os radiográficos.

As principais limitações deste estudo são o fato de ele ser retrospectivo, com quantidade de pacientes relativamente baixa e discrepante entre os grupos.

\section{Conclusão}

Concluímos que o tratamento cirúrgico das LACs apresenta resultados satisfatórios, tanto nos casos agudos quanto nos casos crônicos. Porém, devido à maior tendência a apresentar subluxação residual com aumento do espaço CC nos casos crônicos, devemos buscar tratá-las imediatamente após o trauma.

Conflito de Interesses

Os autores declaram não haver conflito de interesses.

\section{Referências}

1 Arliani GG, Utino AY, Nishimura EM, Terra BB, Belangero PS, Astur DC. Luxação acromioclavicular: tratamento e reabilitação. Perspectivas e tendências atuais do ortopedista brasileiro. Rev Bras Ortop 2015;50(05):515-522 
2 Fukuda K, Craig EV, An KN, Cofield RH, Chao EY. Biomechanical study of the ligamentous system of the acromioclavicular joint. J Bone Joint Surg Am 1986;68(03):434-440

3 Li X, Ma R, Bedi A, Dines DM, Altchek DW, Dines JS. Management of acromioclavicular joint injuries. J Bone Joint Surg Am 2014;96 (01):73-84

4 Williams GR, Nguyen VD, Rockwood CA Jr. Classification and radiographic analysis of acromioclavicular dislocations. Appl Radiol 1989;18(02):29-34

5 Tossy JD, Mead NC, Sigmond HM. Acromioclavicular separations: useful and practical classification for treatment. Clin Orthop Relat Res 1963;28(28):111-119

6 Veado MA, Paiva AA, Pinto MS. Tratamento cirúrgico da luxação acromioclavicular completa. Rev Bras Ortop 2000;35(08): 309-313

7 Domos P, Sim F, Dunne M, White A. Current practice in the management of Rockwood type III acromioclavicular joint dislocations-National survey. J Orthop Surg (Hong Kong) 2017;25(02): 2309499017717868

8 Riand N, Sadowski C, Hoffmeyer P. [Acute acromioclavicular dislocations]. Acta Orthop Belg 1999;65(04):393-403

9 Rockwood CA Jr, Williams GR, Young DC. Injuries to the Acromioclavicular Joint. In: Rockwood CA Jr, Green DP, Buchholz RW, Heckman JD, eds. Fractures in adults. Philadelphia: Lippincott Raven; 1996:1341-1413

10 Galpin RD, Hawkins RJ, Grainger RW. A comparative analysis of operative versus nonoperative treatment of grade III acromioclavicular separations. Clin Orthop Relat Res 1985;(193):150-155

11 Dias JJ, Steingold RF, Richardson RA, Tesfayohannes B, Gregg PJ. The conservative treatment of acromioclavicular dislocation. Review after five years. J Bone Joint Surg Br 1987;69(05):719-722

12 Murena L, Canton G, Vulcano E, Cherubino P. Scapular dyskinesis and SICK scapula syndrome following surgical treatment of type III acute acromioclavicular dislocations. Knee Surg Sports Traumatol Arthrosc 2013;21(05):1146-1150

13 Larsen E, Bjerg-Nielsen A, Christensen P. Conservative or surgical treatment of acromioclavicular dislocation. A prospective, controlled, randomized study. J Bone Joint Surg Am 1986;68(04): 552-555

14 Gallay SH, Hupel TM, Beaton DE, Schemitsch EH, McKee MD. Functional outcome of acromioclavicular joint injury in polytrauma patients. J Orthop Trauma 1998;12(03):159-163

15 Lemos MJ. The evaluation and treatment of the injured acromioclavicular joint in athletes. Am J Sports Med 1998;26(01): 137-144
16 Phemister DB. The treatment of dislocation of the acromioclavicular joint by open reduction and threaded-wire fixation. J Bone Joint Surg Am 1942;24(01):166-168

17 Salzmann GM, Paul J, Sandmann GH, Imhoff AB, Schöttle PB. The coracoidal insertion of the coracoclavicular ligaments: an anatomic study. Am J Sports Med 2008;36(12):2392-2397

18 Grutter PW, Petersen SA. Anatomical acromioclavicular ligament reconstruction: a biomechanical comparison of reconstructive techniques of the acromioclavicular joint. Am J Sports Med 2005; 33(11):1723-1728

19 von Heideken J, Boström Windhamre H, Une-Larsson V, Ekelund A. Acute surgical treatment of acromioclavicular dislocation type $\mathrm{V}$ with a hook plate: superiority to late reconstruction. J Shoulder Elbow Surg 2013;22(01):9-17

20 Rolf O, Hann von Weyhern A, Ewers A, Boehm TD, Gohlke F. Acromioclavicular dislocation Rockwood III-V: results of early versus delayed surgical treatment. Arch Orthop Trauma Surg 2008;128(10):1153-1157

21 Tauber M, Eppel M, Resch H. Acromioclavicular reconstruction using autogenous semitendinosus tendon graft: results of revision surgery in chronic cases. J Shoulder Elbow Surg 2007;16(04): 429-433

22 Hegazy G, Safwat H, Seddik M, Al-Shal EA, Al-Sebai I, Negm M. Modified Weaver-Dunn Procedure Versus The Use of Semitendinosus Autogenous Tendon Graft for Acromioclavicular Joint Reconstruction. Open Orthop J 2016;10:166-178

23 Molin DC, Ribeiro FR, Brasil Filho R, et al. Via de acesso cirúrgico póstero-superior para o tratamento das luxações acromioclaviculares: resultados de 84 casos operados. Rev Bras Ortop 2012;47 (05):563-567

24 Scandiuzzi F, Torquato MT, Mizobuchi RR, Moraes Filho DC, Durigan A Junior. Tratamento cirúrgico da luxação acromioclavicular pela transferência do ligamento coracoacromial. Rev Bras Ortop 1999;34(05):305-312

25 Ferreira Neto AA, Camargo OP, Ferreira Filho AA, et al. Tratamento cirúrgico da luxação acromioclavicular aguda pela técnica de Vukov. Rev Bras Ortop 1996;31:719-726

26 Neviaser JS. Acromioclavicular dislocation treated by transference of the coraco-acromial ligament. A long-term follow-up in a series of 112 cases. Clin Orthop Relat Res 1968;58(58):57-68

27 Horn JS. The traumatic anatomy and treatment of acute acromioclavicular dislocation. J Bone Joint Surg Br 1954;36-B(02):194-201

28 Bjerneld H, Hovelius L, Thorling J. Acromio-clavicular separations treated conservatively. A 5-year follow-up study. Acta Orthop Scand 1983;54(05):743-745 